\title{
Adyuvancia en tratamiento cuirúrgico de fibroma osificante. A propósito de un caso
} Adjuvance in surgical treatment of ossifying fibroma. Case report

Fernando GONZÁLEZ MAGAÑA*, Héctor O. MALAGÓN HIDALGO **, Eugenio GARCíA CANO***,

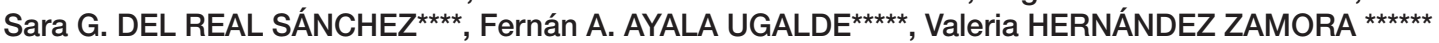

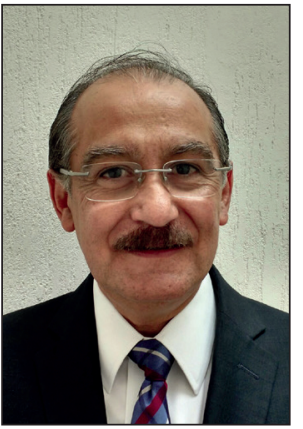

González Magaña F.

\section{Resumen}

El fibroma osificante es una neoplasia osteogénica benigna que representa el $3.1 \%$ de los tumores orales y el $9.6 \%$ de las lesiones gingivales.

$\mathrm{Su}$ tratamiento de elección es la enucleación por curetaje completado hasta márgenes de hueso sano, con una recurrencia de aproximadamente el 7-20\% probablemente por remoción incompleta, irritación continua o daño repetido.

Presentamos un caso clínico en el que complementamos el tratamiento quirúrgico con métodos adyuvantes para disminuir la recurrencia y acelerar la regeneración ósea mediante uso de nitrógeno líquido y hueso liofilizado cadavérico combinados con plasma rico en plaquetas. En el control postoperatorio a los 6 meses no evidenciamos recurrencia clínica o radiológica y comprobamos regeneración ósea acelerada.

Sugerimos el uso en este tipo de lesiones de nitrógeno líquido como adyuvante para la prevención de recurrencia, hueso liofilizado para favorecer la regeneración ósea guiada y plasma rico en plaquetas para acelerar el proceso de curación ósea

\section{Palabras clave Fibroma osificante, Tumores orales, Tumores gingivales}

\section{Nivel de evidencia científica Recibido [esta versión] Aceptado}

4d Terapéutico
29 mayo/2019
5 agosto/2019

Ossifying fibroma is a benign osteogenic neoplasm, representing $3.1 \%$ of oral tumors and $9.6 \%$ of gingival lesions.

Enucleation by curettage is the treatment of choice, completed to healthy bone margins, with a recurrence of approximately $7-20 \%$, probably due to incomplete removal, continued irritation or repeated damage.

We describe a clinical case ttreated in combination with adjuvant methods to decrease recurrence and accelerate bone regeneration, with the placement of liquid nitrogen and cadaveric lyophilized bone, combined with platelet-rich plasma. Post-operative control at 6 months showed no clinical or radiological evidence of recurrence and the presence of accelerated bone regeneration.

We suggest liquid nitrogen as an adjuvant for the prevention of recurrence and bone regeneration guided with lyophilized bone in conjunction with platelet-rich plasma as a feasible option to accelerate the bone healing process.

Conflicto de intereses: Los autores declaran no tener ningún interés financiero relacionado con el contenido de este artículo.

Financiación: No hubo fuentes externas de financiación para este trabajo.

\section{Level of evidence Received [this version] Accepted}

4d Therapeutic

29 May/2019

5 Augost/2019 
Introducción

El fibroma osificante (FO) fue descrito por Menzel en 1872 y su término fue designado por Montgomery en 1927. ${ }^{(1,2)}$ Se trata de una neoplasia osteogénica benigna, sin evidencia de malignización, derivada de células mesenquimatosas multipotenciales del ligamento periodontal y compuesta de tejido fibroso altamente celular con cantidades variables de tejido calcificado que le dan apariencia de hueso, cemento o ambos. ${ }^{(3)}$

Representa el 3.1\% de los tumores orales y el $9.6 \%$ de las lesiones gingivales; de estos, aproximadamente el $60 \%$ se dan en el área maxilar y en el 50\% de estos casos en las áreas de los incisivos y caninos. ${ }^{(4)}$

Generalmente es asintomático hasta que el crecimiento produce aumento de volumen y deformidad, ya que al crecer lentamente no alteran las corticales óseas y la mucosa que las cubre. ${ }^{(5-8)}$

$\mathrm{Su}$ tratamiento consiste en la enucleación o curetaje con un porcentaje de recidiva del 7 al $20 \%$, por lo cual creemos de interés la presentación de un caso clínico en el que empleamos tratamientos adyuvantes con la finalidad de disminuir esta alta tasa de recurrencia, así como para acelerar el proceso de regeneración ósea.

\section{Caso clínico}

Mujer de 43 años de edad sin antecedentes de importancia. Refiere crecimiento en región maxilar derecha desde hace 10 años.

A la exploración física encontramos una lesión de aproximadamente $3 \mathrm{~cm}$ de diámetro a nivel de primero y segundo premolares y primer molar superior derechos en región vestibular y lingual, que ocasiona expansión y deformación del maxilar derecho (Fig. 1).

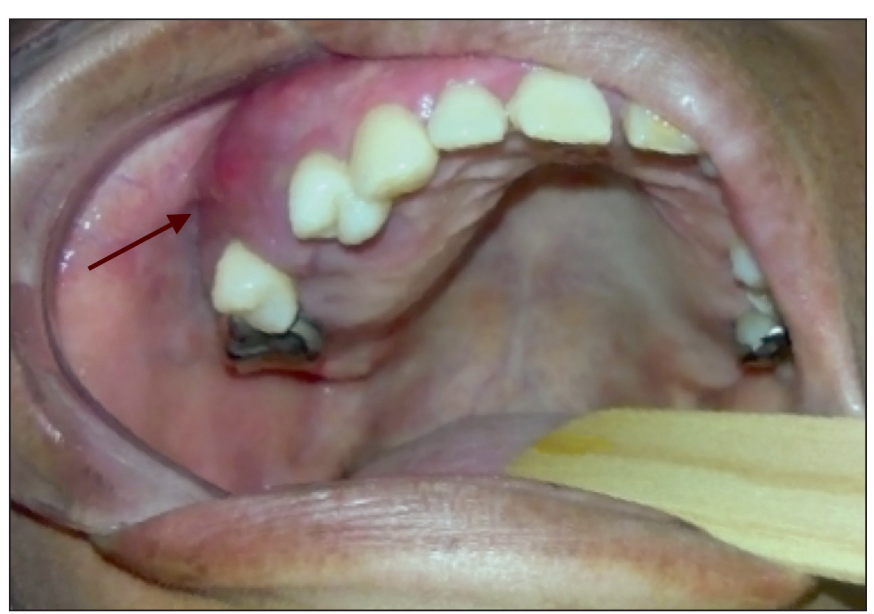

Figura 1. Lesión a nivel de primer y segundo premolares y de primer molar superior derechos en región vestibular y lingual, que ocasiona expansión y deformación maxilar derechas.

En la ortopantomografía observamos una lesión mixta, unilocular, bien delimitada y expansión cortical ósea
(Fig. 2). En la tomografía axial computarizada (TAC), comprobamos que no invade el seno maxilar derecho, presenta bordes bien definidos y densidades mixtas (Fig. 3).

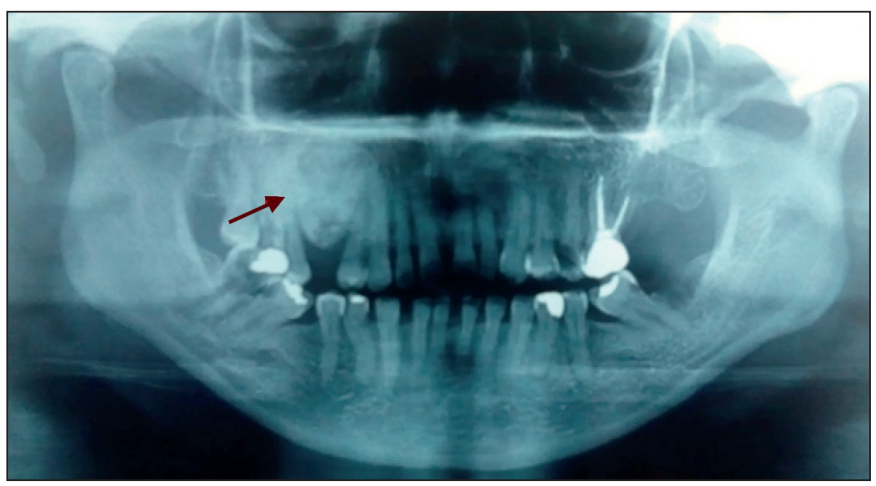

Figura 2. Lesión con densidades mixtas en maxilar derecho, con desplazamiento de piezas dentarias y bordes bien delimitados.

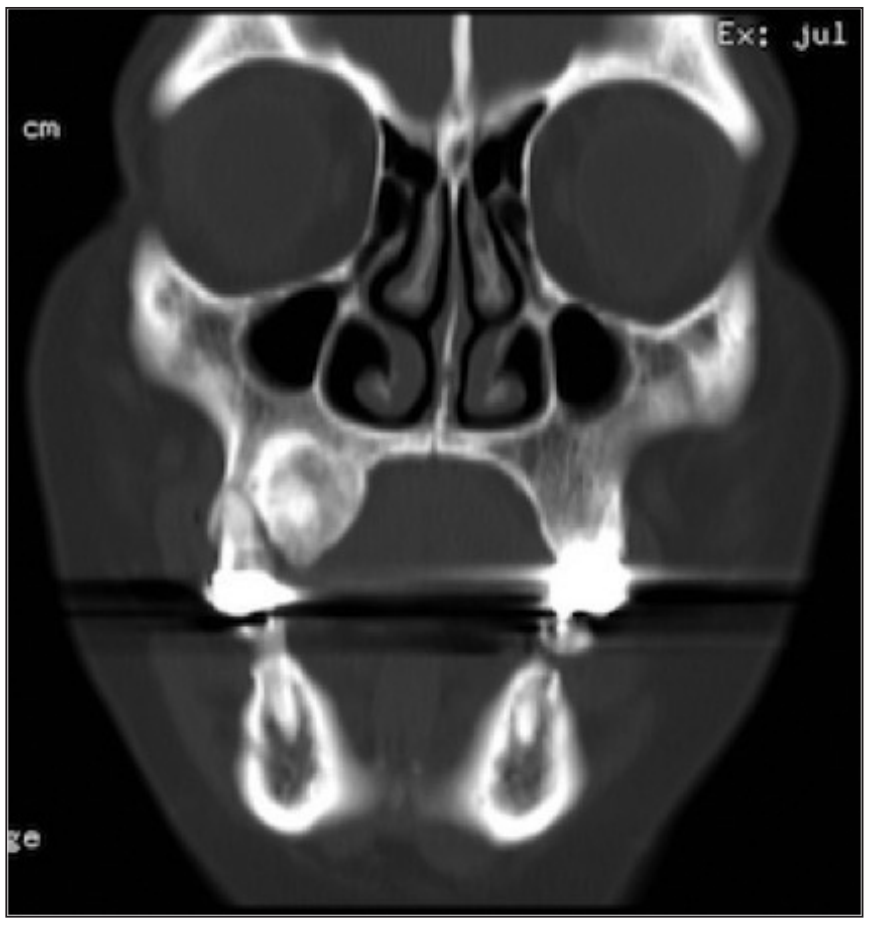

Figura 3. TAC de macizo facial, corte coronal: lesión con densidades mixtas $y$ bordes bien definidos que no invade el seno maxilar ipsilateral.

Realizamos biopsia incisional para conocer la estirpe histopatológica, con resultado de fibroma osificante (FO).

Bajo anestesia general balanceada con intubación nasotraqueal y rutina preoperatoria, practicamos incisión intraoral con colgajo mucoperióstico vestibular y palatino, exponiendo la lesión, y continuando con osteotomías para debilitar la tumoración; posteriormente realizamos enucleación hasta observar márgenes de hueso sano, con curetaje de lecho quirúrgico (Fig. 4).

El defecto creado fue de aproximadamente $3.5 \times 2 \mathrm{~cm}$, con una profundidad aproximada de $1.5 \mathrm{~cm}$. Colocamos en el lecho nitrógeno líquido (NL) a través de un sistema abierto, teniendo cuidado de vesterlo única y exclusiva- 


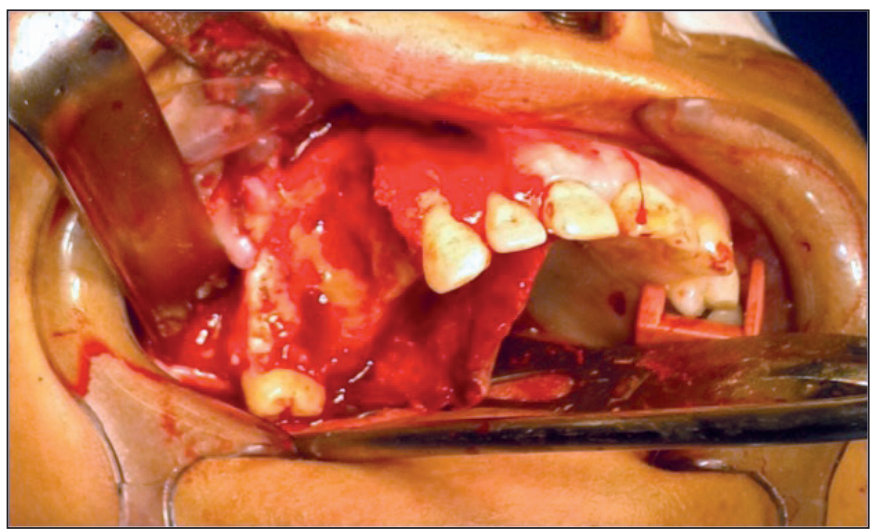

Figura 4. Enucleación completa de la tumoración y curetaje del lecho quirúrgico.

mente en la cavidad creada y lo dejamos actuar durante 5 minutos, posteriormente irrigamos la cavidad con solución salina al $0.9 \%$. Continuamos con la colocación del hueso liofilizado cadavérico (HL) empleando pequeños cuadrados de aproximadamente $5 \times 5 \mathrm{~cm}$ que fuimos posicionando individualmente hasta cubrir por completo del defecto. Combinamos el procedimiento con plasma rico en plaquetas (PRP) obtenido preoperatoriamente de la paciente. Para ello, recolectamos $20 \mathrm{ml}$ de sangre total a través de una vía periférica en 4 tubos estériles con citrato de sodio, centrifugamos a 1.200 revoluciones por minuto (rpm) durante 8 minutos, retiramos un excedente de cada tubo de aproximadamente $2 \mathrm{ml}$ y activamos los $3 \mathrm{ml}$ restantes de plasma de cada tubo con gluconato de calcio en una relación de 1:9 (1 $\mathrm{ml}$ de gluconato de calcio por cada $9 \mathrm{ml}$ de PRP) y colocamos la preparación sobre el injerto de hueso liofilizado cadavérico (Fig. 5). A continuación, reposicionamos el colgajo mucoperióstico con sutura de ácido poliglicólico 3-0.

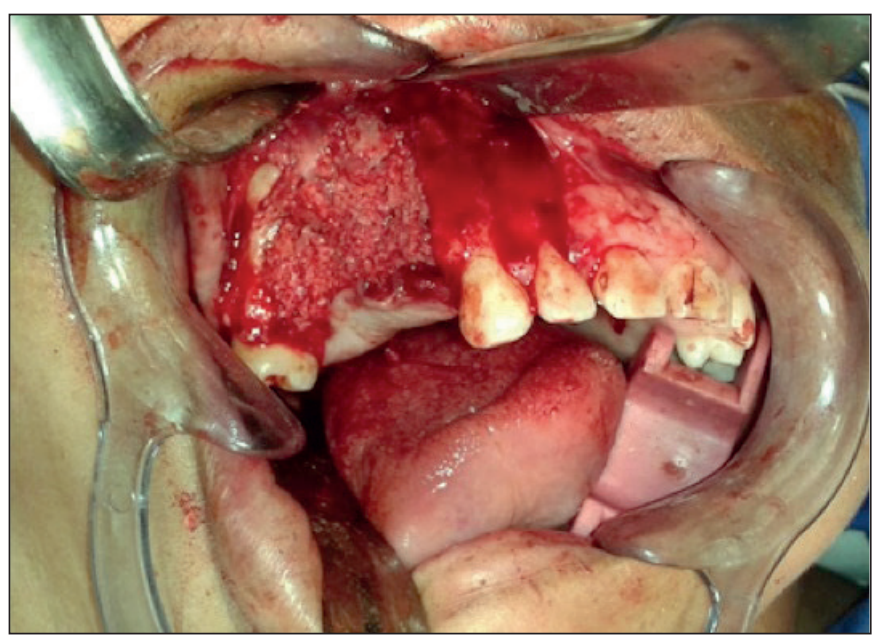

Figura 5. Aplicación de hueso cadavérico liofilizado con plasma rico en plaquetas en el defecto ocasionado por la enucleación.

En el control postoperatorio radiográfico y clínico a los 3 meses de la intervención (Fig. 6 y 7), no hubo evidencia de recurrencia de la tumoración y comprobamos la existencia de regeneración ósea.

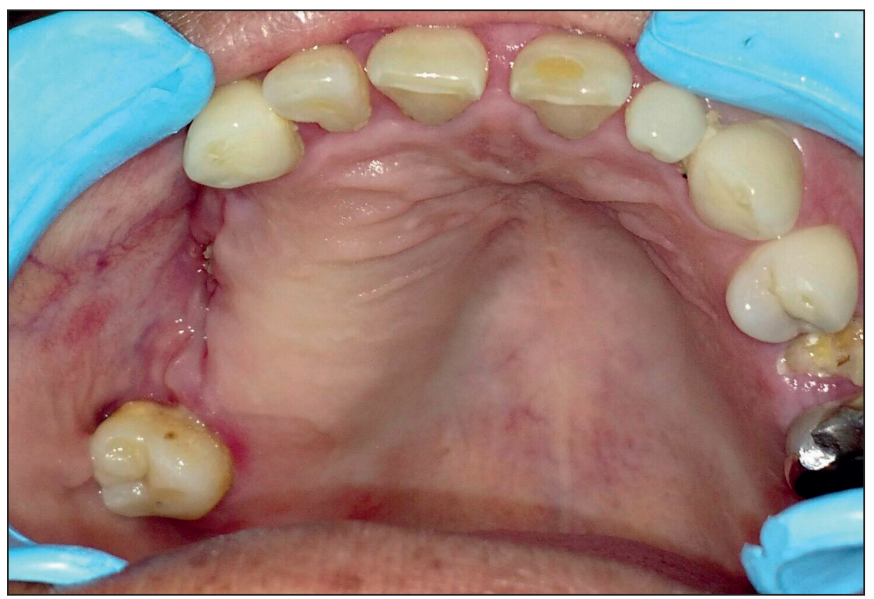

Figura 6. Control clínico a los 3 meses de postoperatorio sin evidencia de recurrencia tumoral en la mucosa.

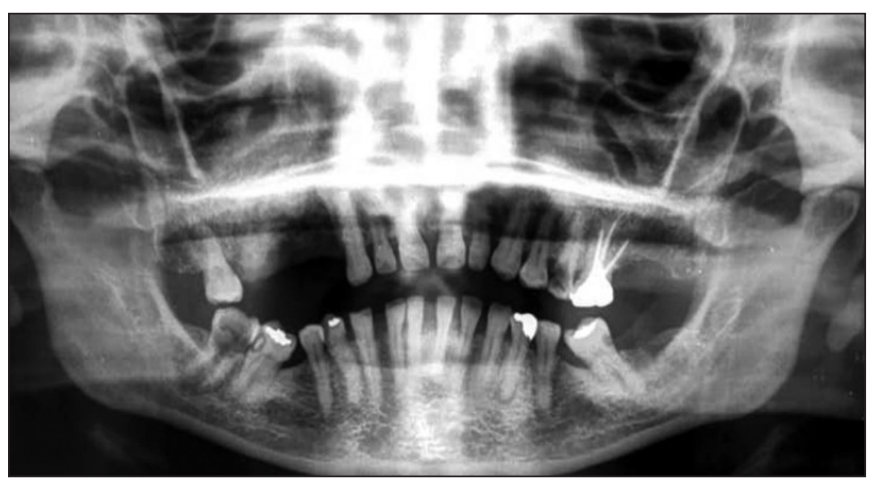

Figura 7. Ortomopantografía de control a los 3 meses de postoperatorio: adecuada regeneración ósea sin evidencia de recurrencia.

\section{Discusión}

El FO tiene su pico de incidencia entre la segunda y tercera décadas de la vida con predilección por el sexo femenino de 5:1, lo cual se corresponde con los datos etarios y de género de nuestra paciente..$^{(9)}$ Como posibles factores desencadenantes se han propuesto traumatismos previos, exodoncias y periodontitis; ${ }^{(5)}$ sin embargo, ninguno de estos factores se presentó en nuestra paciente.

La tumoración se caracteriza por ser indolora, con crecimiento lento, que provoca expansión de las corticales y engrosamiento de las estructuras contiguas, causando deformidad conforme evoluciona dejando que las estructuras que lo cubren estén invariablemente intactas. ${ }^{(3)}$ Un hallazgo notable es su gran tamaño al momento del diagnóstico; nuestra paciente presentaba una deformidad importante por un crecimiento de la lesión de aproximadamente 10 años, sin síntomas concomitantes. Está descrito que, en general, el tamaño de este tipo de lesiones puede variar de 0.2 hasta $15 \mathrm{~cm}$; en nuestro caso, el tamaño de lesión fue de $3 \mathrm{~cm}$ sin llegar a presentar síntomas concomitantes. Radiológicamente se presenta como áreas radiolúcidas con o sin focos radiopacos, bien delimitadas y frecuentemente asociadas con expansión cortical. ${ }^{(10,11)}$ En la ortopantomografía de nuestra paciente 
encontramos una lesión radiolúcida, unilocular, bien delimitada, con expansión cortical. Algunos otros tumores odontogénicos comparten estas características radiológicas, por tal motivo es necesario la confirmación histopatológica para normar el tratamiento definitivo, procedimiento que también seguimos en nuestra paciente.

El tratamiento de elección es la enucleación por curetaje hasta obtener márgenes de hueso sano. Sin embargo, la tasa de recurrencia según la bibliografía es de aproximadamente el $7-20 \%$, probablemente por su remoción incompleta, irritación continúa o daño repetido. ${ }^{(9)}$ Debido a esta elevada tasa de recurrencia, optamos por utilizar tratamientos adyuvantes para, teóricamente, reducirla. Empleamos para ello NL que produce cristales de hielo intracelular con disrupción de la membrana celular y necrosis. Está descrito su uso como adyuvante efectivo en tumores óseos benignos y malignos de bajo grado. ${ }^{(12)}$, razón por la cual decidimos aplicarlo sobre el lecho quirúrgico de resección de nuestro FO, como un adyuvante, para disminuir la recurrencia. En el control radiológico a los 3 meses de la intervención, no observamos recurrencia.

Hasta donde hemos podido comprobar en la literatura no hemos encontrado experiencia reportada sobre el uso de regeneración ósea guiada con hueso liofilizado (ROGHL) para el defecto secundario al tratamiento de este tipo de tumores; sin embargo, Bahadir y col. mencionan su uso para reconstruir la cavidad secundaria a la enucleación de lesiones quísticas odontogénicas gigantes en 17 pacientes, mostrando un fuerte efecto acumulativo con un proceso de curación ósea más rápido. ${ }^{(13)}$ En nuestra paciente, en el control radiológico al tercer mes, pudimos comprobar un proceso de regeneración ósea temprano, con presencia de calcificación y formación trabecular cuando lo normal es esperar verlo entre la cuarta y octava semanas de postoperatorio.

En cuanto al PRP, sabemos que en su forma activa contiene factores de crecimiento derivados de las plaquetas, de crecimiento vascular endotelial, de crecimiento epidérmico, de crecimiento fibroblástico (PDGF, VEGF, EGF, FGF), Factor- $\beta$ (TGF- $\beta$ ), así como macrófagos y monocitos capaces de mediar la inflamación, y está demostrado que su uso tiene ventajas para acelerar la curación ósea, ${ }^{(14)}$ por lo cual lo optamos por aplicarlo de forma empírica en la cavidad secundaria al tratamiento quirúrgico realizado en nuestra paciente, junto con el procedimiento de ROGHL

En nuestra experiencia con el caso presentado, creemos que el ROGHL enriquecido con PRP es la combinación más efectiva cuando se trata de lograr una regeneración ósea más temprana y la curación de tejidos blandos que conduce a una mayor densidad en el tejido óseo maduro. ${ }^{(15)}$
Conclusiones

El fibroma osificante de la maxila es un tumor benigno raro, cuyo tratamiento quirúrgico de primera instancia debe ser conservador con enucleación completa y curetaje del lecho quirúrgico para prevenir la recurrencia.

Proponemos el uso como terapia coadyuvante a la cirugía en este tipo de tumores de nitrógeno líquido para prevenir la recidiva tumoral y el método de regeneración ósea guiada por hueso liofilizado en conjunto con plasma rico en plaquetas para acelerar el proceso de regeneración ósea.

\section{Dirección del autor}

\author{
Dr. Fernando González Magaña \\ Centro Médico ISSEMYM Toluca \\ Avda. Baja Velocidad 284 \\ San Jerónimo Chicuacalco, Metepec \\ Estado de México, México CP 52170 \\ Correo electrónico: fergonzalez_max@hotmail.com
}

\section{Bibliografía}

1. Huebner GR, Brenneise CV, Ballenger J. Central ossifying fibroma of the anterior maxilla: report of case. J Am Dent As Soc 1988; 116: 507-510.

2. Walter JM, Terry BC, Small EW, Matteson SR, Howell RM. Aggressive ossifying fibroma of the maxilla: review of the literature and report of case. J Oral Surg 1979; 37: 276-286.

3. Vura NG, Gaddipati R, Ramisetti S, Kumara R, Reddy R, Kanchi U. Surgical Management of Ossifying Fibroma in Maxilla: Report of Two cases. J Int Oral Health. 2015;7(3):1-4.

4. Batra J, Kumar P, Chahar M, Attresh G, Berwal V. Management of Massive Peripheral Ossifying Fibroma in the Right Lingual Vestibule of Mandible. J Dent App. 2015;2(6):243-245.

5. Martín-Granizo R, Sánchez-Cuellar A, Falahat F. Cemento ossifying fibroma of the upper gingivae. Otolaryngol Head Neck Surg 2000;122:775.

6. Zachariades N, Vairaktaris E, Papanicolau S, Triantafyllou D, Papavassiliou D, Mezitis M. Ossifying fibroma of the jaws. Review of the literature and report of 16 cases. Int J Oral Surg 1984;13:1

7. Aguirre JM. Tumores de los maxilares. En: Bagán JV, Ceballos A, Bermejo A, Aguirre JM, Peñarrocha M. Medicina Oral. Barcelona: Masson; 1995.Pp. 507-508.

8. Sapp JP, Eversole LR, Wysocki GP. Patología oral y maxilofacial contemporánea. Madrid: Hartcourt Brace España; 1998. Pp. 109-110.

9. Nazareth B, Arya H, Ansari S, Arora R. Peripheral Ossifying Fibroma - A Clinical Report. Int J Odonto Stomat. 2011;5(2):153-156.

10. Su L, Weathers DR, Waldron CA. Distinguishing features of focal cement osseous dysplasia and cement ossifying fibromas II. A clinical and radiologic spectrum of 316 cases. Oral Surg Oral Med, Oral Pathol, Oral Radiol Endod. 1997; 84: 540-549.

11. Eversole LR, Merrell PW, Strub D. Radiographic characteristics of central ossifying fibroma. Oral Surg, Oral Med, Oral Pathol. 1985; 59: 522-527.

12. Bickels J, Meller I, Shmookler BM, Malawer MM. The role and biology of Cryosurgery in the treatment of Bone Tumors: A review. Acta Orthop Scand. 1999;70(3):306-315.

13. Bahadir G, Gokce M. Allogenic Grafts in Oral Surgery: Clinical Findings and Follow-up. Hacettepe Dishekimligi Fakultesi Dergisi 2007. 31(2):31-37.

14. Rodríguez IA, Growney-Kalaf EA, Bowlin GL, Sell SA. Platelet-Rich Plasma in Bone Regeneration: Engineering the Delivery for Improved Clinical Efficacy. Bio Med Research Int. 2014;2014:392398. doi: http://dx.doi. org/10.1155/2014/392398

15. Sethi AK, Kar IB, Mohanty T, Mishra N, Singh AK. Use of plasma-enriched demineralized freeze-dried bone matrix in postsurgical jaw defects. Natl J Maxillofac Surg. 2018;9(2):174-183. 